\title{
PROTEOMICS
}

\section{RNA-binding proteome redux}

Scientists exploit the basic physicochemical properties of protein-RNA complexes to paint a dynamic portrait of the RNA-binding proteome.

S ubstantial efforts have been directed toward defining the full extent of the RNA-binding proteome (RBPome) over the past decade. Most of those efforts have relied on the cross-linking of protein to RNA followed by sequence-specific isolation of the resulting complexes.

Although this method has proved successful at providing large repertoires of RNAbinding proteins (RBPs), drawbacks include the need for sizable amounts of material and complex workflows.

To address these limitations, a group from the Cambridge Center for Proteomics, led by Kathryn Lilley, Eneko Villanueva, Tom Smith, and Rayner Queiroz, took advantage of the well-known tendency of proteins and RNA to partition into organic and aqueous solvents, respectively. The scientists demonstrated that once proteins were cross-linked to RNA by UV irradiation, the protein-RNA complexeswhich combine the properties of each of their individual components-could be specifically and efficiently isolated from the aqueous-organic interface for downstream analysis by mass spectrometry and RNA sequencing. The approach is termed OOPS, short for "orthogonal organic phase separation."

Analysis of the protein and RNA components isolated by OOPS demonstrated that both coding and noncoding RNAs with sizes greater than 60 bases are isolated without bias, and the RNA-binding sites could be inferred from the lack of sequence coverage caused by cross-linking at the point of interaction. The group also used OOPS to define a first bacterial protein-RNA interactome. Finally, they used OOPS to document dynamic changes in RNA-binding patterns during cell cycle progression in human cells.

Because of its simplicity and relatively reduced material requirements, OOPS promises not only to facilitate the cataloging of protein-RNA interactions, but also to provide a system-wide view of the RBPome by enabling studies of its dynamics.

Stéphane Larochelle

Published online: 27 February 2019

https://doi.org/10.1038/s41592-019-0349-3

\section{Research papers}

Queiroz, R. M. L. et al. Comprehensive identification of RNA-protein interactions in any organism using orthogonal organic phase separation (OOPS). Nat. Biotechnol. 37, 169-178 (2019).

\section{xboc COMMUNICATIONS BIOLOGY}

\section{NOW PUBLISHING CONTENT}

\section{A new open access journal for the biological sciences from Nature Research}

Communications Biology publishes high-quality primary research articles, reviews and commentary in all areas of the biological sciences. Papers published in the journal represent significant advances that bring new insight to a specialized area of research.

All papers are handled by experienced in-house professional editors supported by an expert Editorial board.

\section{Submit your research today and benefit from:}

- Thorough peer review

- Fast decision process

- High Nature editorial standards
- High visibility

- $\quad$ CC-BY open access as standard

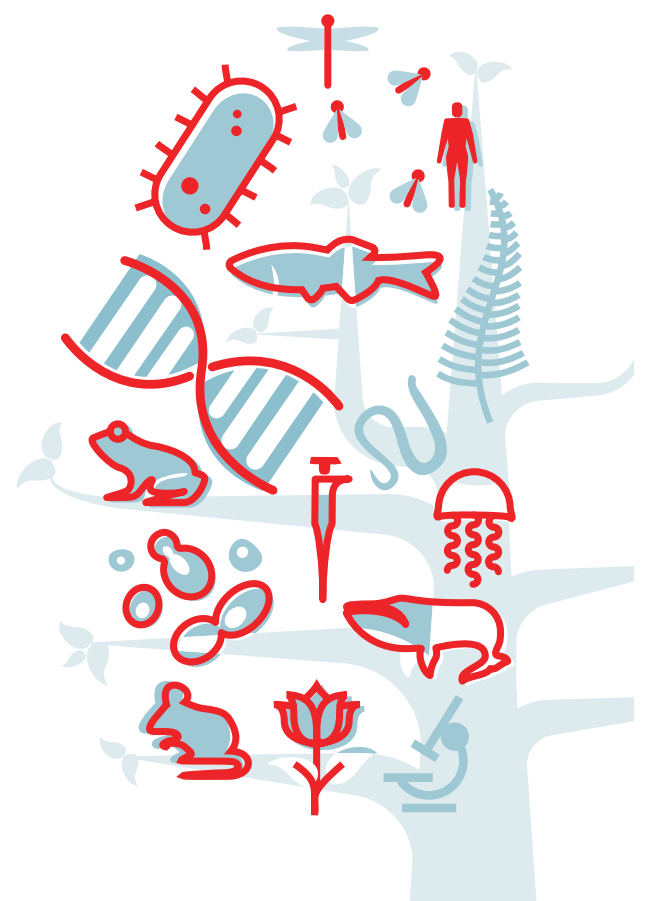

